\title{
TOLERÂNCIA DE JUVENIS DE PACU PIARACTUS MESOPOTAMICUS À ÁGUA SALINIZADA
}

\author{
BARBOSA JÚNIOR, Sérgio Garcia ${ }^{1}$ \\ TEIXEIRA, Regiane Batista Gonçalves ${ }^{2}$ \\ CABRAL, Marina Calil ${ }^{2}$ \\ ASSANO, Marcelo ${ }^{3}$ \\ JOMORI, Rosangela Kiyoko ${ }^{4}$
}

Recebido em: 2010.09.15 Aprovado em: 2010.10.29

ISSUE DOI: $10.3738 / 1982.2278-467$

\begin{abstract}
RESUMO: O cloreto de sódio é um produto comumente utilizado durante a criação de peixes de água doce em manejos profiláticos e terapêuticos. O objetivo do trabalho foi avaliar o desempenho e o tempo de tolerância de juvenis de pacu $P$. mesopotamicus submetidos a diferentes salinidades da água: 0 (água doce), 3, 6, 9, 12, 15 $\%$, com três repetições, durante 20 dias. $\mathrm{O}$ experimento foi realizado em unidades contendo 12 litros de água cada, em sistema de água parada e substituição diária de 100\% do volume. Em outro experimento, determinou-se o tempo de tolerância dos juvenis em concentrações de $10,15,20,30,40$ e $50 \%$, por períodos de 15,30 e 45 minutos de exposição. Posteriormente, nas salinidades de 30, 40 e 50\%, o tempo de tolerância foi investigado por períodos de 4,8 e 12 minutos. Não houve diferenças $(P>0,05)$ para o crescimento entre os animais mantidos nas salinidades de 0 a $9 \%$. Já nas salinidades de 15 e 12\%o, observou-se a mortalidade total (100 \%) após 6 e 24 horas de experimento, respectivamente. Nas salinidades mais elevadas, de 30, 40 e $50 \%$, observou-se 66, 100 e $100 \%$ de mortalidade nos tempos de 15, 30 e 45 minutos de exposição, respectivamente. Por outro lado, a permanência dos animais nas concentrações de 30, 40 e 50\%o por períodos de 4, 8 e 12 minutos não causou mortalidade dos peixes, e $100 \%$ dos animais sobreviveram após o retorno para a água doce.
\end{abstract}

Palavras-chave: Cloreto de sódio. Salinidade da água. Sobrevivência.

SUMMARY: The sodium chloride is a product commonly used in the rearing of freshwater fish in prophylaxis and treatment therapeutic. The objective of this work was to evaluate the survival and the time of tolerance of juvenile of pacu Piaractus mesopotamicus in different salinity of water: 0 (freshwater), 3, 6, 9, 12 and $15 \%$, with 3 replicates, during 20 days. The experiment was carried out in tanks containing $12 \mathrm{~L}$ of water in each, in systems of rested water with the daily change of $100 \%$ of capacity. In the second experiment, the tolerance time was determinated at 10,15, 20,30, 40 and $50 \%$ of salinity during 15,30 and 45 minutes. In the salinities of 30, 40 and $50 \%$, the tolerance time was also evaluated for the periods of 4,8 and 12 minutes. There was no difference $(\mathrm{p}>0.05)$ among the averages of length and weight of the animals kept in salinities of 0 to $9 \%$. The total mortality (100\%) occurred at 15 and $12 \%$ after 6 and 24 hours of experiment, respectively. In the second experiment, to the animals kept at 30,40 and 50\%o, it was observed 66, 100 and $100 \%$ of mortality in periods of 15,30 and 45 minutes of exhibition, respectively. But, the concentrations of 30,40 and $50 \%$ for periods of 4, 8 and 12 minutes did not cause fish`s mortality, and 100\% of animals survived after their transfer to freshwater.

Keywords: Sodium chloride. Water Salinity. Survival

\footnotetext{
${ }^{1}$ Graduando do Curso de Medicina Veterinária da Faculdade Dr Francisco Maeda, FAFRAM/ FE, Ituverava, SP.

${ }^{2}$ Graduanda do Curso de Biologia da Eaculdade de Filosofia, Ciências e Letras, FFCL/ FE, Ituverava, SP. ${ }^{2}$ Bolsista do Centro de Integração Empresa Escola (CIEE).

${ }^{3}$ Zootecnista, Mestre em Aquicultura

${ }^{4}$ Professora Doutora da Faculdade Dr Francisco Maeda, Laboratório de Aquicultura, FAFRAM/ FE Ituverava, SP. Pós-Doutoranda do Centro de Isótopos Estáveis, IB/UNESP, Botucatu, SP. Autor Correspondente: jomori@netsite.com.br
} 


\section{INTRODUÇÃO}

A criação comercial de peixes no mundo é uma atividade que vem se tornando cada vez mais importante para o fornecimento de fonte protéica de qualidade para consumo humano. Durante a criação, os peixes são expostos a agentes agressores, e estes procedimentos são inevitáveis nas atividades de uma piscicultura (URBINATI; CARNEIRO, 2004), e um dos produtos recomendados para ações profiláticas e no combate a determinadas enfermidades é o cloreto de sódio (PAVANELLI; EIRAS; TAKEMOTO, 1998; ALTINOK; GRIZZLE, 2001b), o sal comum utilizado para fins agropecuário.

Em situações adversas, acentuam-se as perdas de sais do sangue para a água (CARNEIRO; URBINATI, 2004) e a adição de cloreto de sódio na água de criação dos peixes minimiza a diferença osmótica entre o animal e o meio externo, e também estimula a secreção de muco sobre o epitélio branquial, dificultando a passagem dos íons através das membranas celulares (WURTS, 1995).

As concentrações de sal para uso em banhos profiláticos e terapêuticos variam conforme a idade do animal, o tempo de duração dos banhos e o agente patológico (PAVANELLI; EIRAS; TAKEMOTO., 1998).

O pacu Piaractus mesopotamicus é uma espécie nativa de água doce com ampla distribuição pelos rios que compõem a Bacia do Prata. A espécie destaca-se pela sua rusticidade ao manejo e possui grande potencial para a aqüicultura (VAZ; TORQUATO; BARBOSA, 2000; PROENÇA; BITTENCOURT, 1994). Desta forma, o objetivo do estudo foi avaliar o desempenho e a tolerância de juvenis de pacu submetidos a diferentes meios salinizados

\section{MATERIAL E MÉTODOS}

O estudo foi realizado na Faculdade "Dr Francisco Maeda" - FAFRAM/ FE, Ituverava/ SP, no Laboratório de Aqüicultura. Para a realização dos experimentos foram utilizados juvenis de pacu Piaractus mesopotamicus, em fase inicial de crescimento, apresentando médias de comprimento total e peso de $10,9 \pm 0,3 \mathrm{~cm} \mathrm{e} 22,5 \pm 2,4 \mathrm{~g}$, respectivamente. Foram estudadas duas situações de exposição a ambientes salinizados: a manutenção dos juvenis em ambientes levemente salinizados (Experimento I) por um período de 20 dias, e a exposição a concentrações mais elevadas por intervalos de até 45 minutos (Exp. II). 
No experimento I, os animais foram submetidos às salinidades de 0 (água doce), 3, 6, 9, 12 e $15 \%$, durante 20 dias, seguido pela transferência direta dos animais para a água doce, com 5 dias de observação. O experimento foi conduzido em delineamento inteiramente casualizado, com três repetições por tratamento, e foram avaliados o crescimento dos animais (em comprimento e peso) e a taxa de sobrevivência.

Os juvenis ( $n=4$ em cada repetição) foram estocados em aquários contendo $12 \mathrm{~L}$ de água cada, em sistema de banho termostatizado para a manutenção da temperatura em cerca de $28^{\circ} \mathrm{C}$, com aeração artificial e renovação diária da água em cerca de $100 \%$ do volume total.

Os peixes foram alimentados uma vez ao dia, com ração comercial contendo $42 \%$ de proteína bruta, e o alimento foi oferecido até a saciedade aparente, com o controle diário do alimento consumido.

No segundo experimento, os animais foram mantidos nas salinidades de 20, 30, 40 e $50 \%$ o, tendo a salinidade de $10 \%$ o como controle; e com base nas respostas do Experimento I, repetiu-se a avaliação da tolerância dos peixes a $15 \%$. Os animais (n = 9 para cada salinidade) foram mantidos nestas concentrações de sal por tempos de 15, 30 e 45 minutos e, em seguida, transferidos para a água doce. Os animais foram mantidos em aquários contendo 12 L de água, com aeração artificial. Com base nos resultados desta avaliação, outros animais foram mantidos nas salinidades de 30, 40 e $50 \%$ o com o tempo de exposição menor: de 4, 8 e 12 minutos, seguidos pela transferência para a água doce. Os juvenis $(n=6$ para cada salinidade) foram acondicionados em unidades experimentais contendo $5 \mathrm{~L}$ de água cada, com aeração artificial. Nestas duas avaliações, o tempo de tolerância dos animais foi determinado pela ocorrência da mortalidade dos peixes nos meios salinos, e pela sobrevivência dos animais após serem transferidos para a água doce, com um período de observação de 24 horas.

Os valores médios de comprimento total, peso e taxa de sobrevivência, obtido em cada réplica experimental (Exp. I), foram analisados estatisticamente através da análise de variância paramétrica (ANOVA). As taxas de sobrevivência sofreram transformação arco seno. Nos resultados em que foram verificadas diferenças significativas entre tratamentos $(\mathrm{p}<0,05)$, as médias foram comparadas pelo teste Tukey, ao nível de 5\% de probabilidade.

\section{RESULTADOS E DISCUSSÃO}

Ao final de 20 dias, não foram verificadas diferenças $(\mathrm{P}>0,05)$ para as médias de comprimento e peso dos juvenis de pacu mantidos nas salinidades em até $9 \%$ (Tabela 1). 
TABELA 1 - Analise de variância pelo teste F e valores médios de comprimento $(\mathrm{cm})$ e peso (g) de juvenis de pacu Piaractus mesopotamicus submetidos a diferentes salinidades, após 20 dias de exposição.

\begin{tabular}{ccc}
\hline \multirow{2}{*}{ Estatística } & \multicolumn{2}{c}{ Valores de F } \\
\cline { 2 - 3 } & Comprimento total & Peso \\
\hline Salinidade (S) & $1,13^{\text {NS }} 1,95^{\text {NS }}$ \\
CV (\%) & 5,60 & 17,31 \\
\hline $\begin{array}{c}\text { Médias para a } \\
\text { salinidade de (\%): }\end{array}$ & $10,3 \pm 7,1$ & $20,4 \pm 4,1$ \\
0 & $9,8 \pm 2,7$ & $17,2 \pm 1,2$ \\
6 & $10,2 \pm 1,6$ & $18,1 \pm 2,6$ \\
9 & $10,2 \pm 1,3$ & $18,0 \pm 5,2$ \\
\hline
\end{tabular}

A taxa de sobrevivência foi $100 \%$ na água doce e na salinizada a $6 \%$, e $83 \pm 14 \%$ a 9\%o $(\mathrm{P}>0,05)$. Contudo, o resultado da taxa de sobrevivência foi prejudicado no final do experimento principalmente para a água salinizada a 3\%o, em que foi observada uma taxa de $68 \pm 28 \%(\mathrm{P}<0,05)$. Próximo de completar os 20 dias, houve uma falha no termostato dos aquecedores provocando um aquecimento excessivo na água, em duas réplicas experimentais da salinidade de $3 \%$ e uma da salinidade de $9 \%$. Alguns exemplares morreram nestas repetições com sintomas da "doença da coluna" causada pela Flexibacter columnaris. Podese considerar que a mortalidade dos juvenis nestas réplicas não foi causada pela concentração de sal a $3 \%$ o. O fato pode ser corroborado pelo resultado obtido na salinidade superior, em que os peixes adaptaram e mostraram-se tolerantes a $6 \%$, com $100 \%$ de sobrevivência em todas as réplicas experimentais. O sangue do peixe tem uma salinidade próxima a 9\%o, e cerca de 77\% dos sais no sangue são sódio e cloreto (WURTS, 1998). Para juvenis de tambaqui Colossoma macropomum, que é uma espécie próxima ao pacu, ambos pertencentes à família Characidae, Gomes et al. (2003) observaram que a concentração de 8 \%o foi eficiente para diminuir a maioria das respostas fisiológicas ao estresse durante o transporte dos animais. Camargo, Pouey e Vaz (2006) também constataram que a espécie jundiá Rhamdia quelen adaptou-se bem a concentração de $8 \%$, até 30 dias de exposição.

Com a mesma espécie pacu $P$. mesopotamicus, na fase de desenvolvimento larval, Jomori, Luz e Portella (2003) observaram que os animais, com nove dias de vida, adaptaram- 
se às salinidades de até $4 \%$, com 100 e $93 \%$ de sobrevivência a 2 e $4 \%$. Contudo, a amplitude da faixa de salinidade suportada pelas larvas foi menor que a observada para os juvenis, com 61 e $100 \%$ de mortalidade das larvas de pacu a 8 e $10 \%$, respectivamente. A faixa de tolerância dos peixes à água salinizada depende de características como a espécie, a idade e o tamanho do animal (WATANABE et al., 1985; ALTINOK; GRAZZLE, 2001a; FASHINA-BOMBATA; BUSARI, 2003). O aumento da tolerância dos peixes à salinidade conforme a idade também foi verificada para larvas de pacamã $L$. alexandri, recomendandose salinidades de até 4\%o para larvas recém-eclodidas e salinidades de até 6\%o para larvas com 8 e 12 dias de vida (LUZ; SANTOS, 2008).

Já nas salinidades de 12 e $15 \%$, a mortalidade total (100\%) ocorreu em todas as repetições, num período de 6 a 20 horas de exposição na salinidade de $15 \%$ e em cerca de 24 a 68 horas, a $12 \%$. A diminuição da taxa de sobrevivência com o aumento da salinidade é um comportamento de peixes estenoalinos de água doce (FASHINA-BOMBATA; BUSARI, 2003). Segundo os autores, exemplares de Heterobranchus longifilis (com 28 dias de vida) apresentaram, no quarto dia de observação, uma sobrevivência de $100 \%$ entre as salinidades de zero a 6\%o. A partir dessa concentração, a taxa de sobrevivência diminuiu alcançando uma média de $20 \%$ na salinidade de 7,5\%o e mortalidade total nas concentrações de 9 e $15 \%$.

Após 20 dias de experimento, os juvenis de pacu adaptaram-se bem às mudanças de concentração osmótica da água e não houve mortalidade com a transferência direta dos juvenis para a água doce.

$\mathrm{Na}$ avaliação do tempo de tolerância às elevadas concentrações de sal (segundo experimento), a taxa de sobrevivência obtida a 10, 15 e $20 \%$ de salinidade foi $100 \%$ em todos os tempos avaliados, em 15, 30 e 45 minutos de exposição (Figura 1). Nas salinidades superiores (30, 40 e $50 \%$ ), observou-se $100 \%$ de mortalidade nos tempos de 30 e 45 minutos. Os peixes não suportaram tais concentrações por estes períodos e não se recuperaram após serem transferidos novamente para a água doce. Com 15 minutos de permanência na salinidade de $30 \%$ não houve mortalidade, mas nas salinidades de 40 e 50\%o, a taxa de mortalidade foi considerável, cerca de $66 \%$. Na salinidade de $50 \%$, observou-se que logo após os primeiros 3 minutos de exposição, os juvenis começaram a apresentar uma natação errática, mantendo-se na superfície da água. 


\section{Taxa de sobrevivência $(\%)$}

\section{Tempo de Exposição}

$\square 15$ min. $\quad$ $30 \mathrm{~min} . \quad \square 45 \mathrm{~min}$.

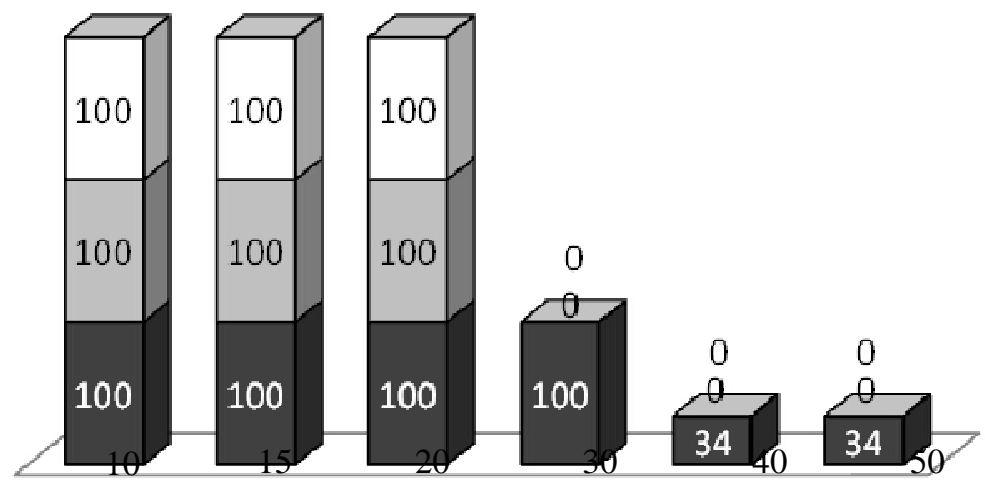

Salinidade $(\%)$

Figura 1. Taxa de Sobrevivência de juvenis de pacu $P$. mesopotamicus submetidos a diferentes salinidades por tempos de 15, 30 e 45 minutos.

Entretanto, a permanência dos animais nas concentrações de 30, 40 e 50 \%o por intervalos menores, períodos de 4, 8 e 12 minutos de exposição, não comprometeu a sobrevida dos juvenis. Os juvenis se recuperaram após o retorno para a água doce (Figura 2), mesmo tendo expressado o comportamento da natação errática e desorientada na superfície da água, e houve $100 \%$ de sobrevivência em todas as concentrações elevadas. Estas respostas evidenciam que a faixa de tolerância em tempo está intimamente correlacionada com a concentração sal, e uma pequena variação neste tempo de exposição é decisiva para permitir à sobrevida ou causar à morte dos juvenis, principalmente quanto mais elevada for a concentração de sal na água. Outras pesquisas nesta linha de investigação estão em andamento visando complementar e corroborar as informações obtidas neste estudo, principalmente quanto à tolerância a salinidades mais elevadas. 
Taxa de sobrevivência (\%)

\section{Tempos de Exposição \\ $\square 4 \mathrm{~min} . \quad \square 8 \mathrm{~min} . \quad \square 12 \mathrm{~min}$}

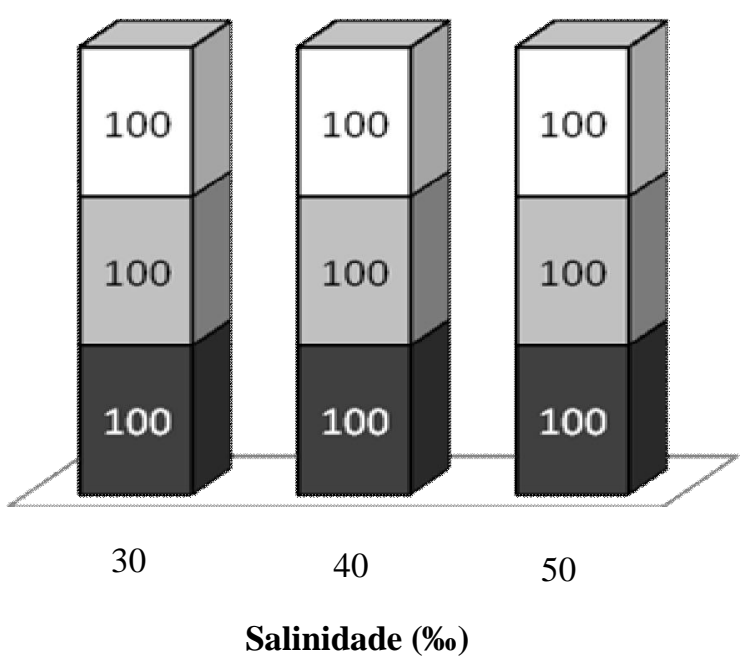

Figura 2. Taxa de Sobrevivência de juvenis de pacu $P$. mesopotamicus submetidos a diferentes salinidades por tempos de 4,8 e 12 minutos de exposição.

\section{CONCLUSÃO}

Os juvenis de pacu mostraram-se capazes de se adaptar às salinidades de até $6 \%$, sem prejuízos à sobrevivência, mesmo quando as mudanças na concentração osmótica da água procederam-se diretamente, sem alteração gradual.

Os juvenis de pacu, em fase de crescimento inicial, toleraram uma exposição contínua na concentração de sal de até $20 \%$ o por um período de até 45 minutos, sem prejuízos à sobrevivência.

Em elevadas concentrações de sal, acima de $30 \%$, os juvenis de pacu não suportaram um tempo de exposição acima de 15 minutos.

\section{REFERÊNCIAS}

ALTINOK, I.; GRIZZLE, J.M. Effects of low salinities on Flavobacterium colummare infection of euryhaline and and freshwater stenohaline fishes. J. Fish Diseases, Edinburgh, v.24, n.6, p.361-367, 2001a. 
ALTINOK, I.; GRIZZLE, J.M. Effects of brackish water on growth, feed conversion and energy absorption efficiency by juveniles euryhaline and freshwater stenohaline fishes. J. Fish Biol., Amsterdam, V.59, n.5, p.1152, 2001b.

CAMARGO, S. G. O.; POUEY, J. L. O. F.; VAZ, B. S. Efeito da salinidade nos parâmetros hematologicos do Jundiá. Revista Brasileira de Agrociência. v. 12 , n. 4, p 453-460. 2006.

CARNEIRO, P. C. F.; URBINATI, E. C. As a stress response mitigator of matrinxã Brycon cephalus (Teleostei: Characoidei) during transport. Aquaculture Research, v. 32, n.4, p.298307, 2001.

FASHINA-BOMBATA, H. A.; BUSARI, A.N. Influence of salinity on the developmental stages of African catfish Heterobranchus longifilis (Valenciennes, 1840). Aquaculture, Amsterdam, v.224, n.1-4, p.213-222, 2003.

GOMES, L.C.et al. Avaliação dos efeitos da adição de sal e da densidade no transporte de tambaqui. Pesquisa agropecuária brasileira. v.38, n.2, p.283-290. 2003.

JOMORI, R.K.; LUZ, R.K.; PORTELLA, M.C. The effect of salinity and growth and survival of pacu Piaractus mesopotamicus larvae. In: WORLD AQUACULTURE, 2003,

Salvador/Bahia. Book of Abstracts... Salvador/Bahia, 2003. p.382.

LUZ, R. K.; SANTOS, J. C. E. Avaliação da tolerância de larvas do pacamã Lophiosilurus alexandri Steindachner, 1877 (Pisces: Siluriformes) a diferentes salinidades. Acta. Sci. Biol. Sci. v. 30, n. 4, p 345-350. 2008.

PAVANELLI, G.C.; EIRAS, J.C., TAKEMOTO, R.M. Doenças de peixes: profilaxia, tratamento e diagnóstico. Maringá: EDUEM, Nupélia, p. 264, 1998.

PROENÇA, C.E.M.; BITTENCOURT, P.R.L. Manual de piscicultura tropical. 1994. p 195.

URBINATI, E.C.; CARNEIRO, P. C. F. Práticas de manejo e estressse dos peixes em piscicultura. In: POSSEBON, J. E. (ed.). Tópicos especiais em piscicultura de água doce. São Paulo. 2004. 533p.

VAZ, M.M.; TORQUATO, V.C.; BARBOSA, N.D.C. Guia ilustrado de peixes da bacia do Rio Grande. 2000. p. 141.

WATANABE, W.O. et al. The ontogeny of salinity tolerance iin the tilapias Oreochromis aureus, $O$. niloticus, and on $O$. mossambicus $x$ O. niloticus hybrid, spawned and reared in frswater. Aquaculture, Amsterdam, v.47, n.4, p.353-367, 1985.

WURTS, W.A. Using salt to reduce handling stress in channel catfish. World aquaculture, Lousiana, v.26, n.3, p.80-81, 1995.

WURTS, W.A. Why can some fish live in freshwater, some in salt water, and some in both? World Aquaculture, Lousiana, v. 29, n.1, p. 65, 1998. 